Photoacoustic imaging with planoconcave optical microresonator sensors: feasibility studies based on phantom imaging

James A. Guggenheim Edward Z. Zhang Paul C. Beard 


\title{
Photoacoustic imaging with planoconcave optical microresonator sensors: feasibility studies based on phantom imaging
}

\author{
James A. Guggenheim, Edward Z. Zhang and Paul C. Beard \\ Department of Medical Physics and Biomedical Engineering, University College London, Gower \\ Street, London WC1E 6BT, UK.
}

\begin{abstract}
The planar Fabry-Pérot (FP) sensor provides high quality photoacoustic (PA) images but beam walk-off limits sensitivity and thus penetration depth to $\approx 1 \mathrm{~cm}$. Planoconcave microresonator sensors eliminate beam walk-off enabling sensitivity to be increased by an order-of-magnitude whilst retaining the highly favourable frequency response and directional characteristics of the FP sensor. The first tomographic PA images obtained in a tissuerealistic phantom using the new sensors are described. These show that the microresonator sensors provide near identical image quality as the planar FP sensor but with significantly greater penetration depth (e.g. $2-3 \mathrm{~cm})$ due to their higher sensitivity. This offers the prospect of whole body small animal imaging and clinical imaging to depths previously unattainable using the FP planar sensor.
\end{abstract}

\section{INTRODUCTION}

The thin film polymer Fabry-Pérot (FP) sensor based photoacoustic (PA) imaging system [1] shown in figure 1 is one of several non-traditional ultrasound detection systems based on optical rather than piezoelectric detection schemes. Other such systems include those based on micro-ring resonators [2], fiber Bragg gratings [3], CCDs [4] and probe beam deflection [5].

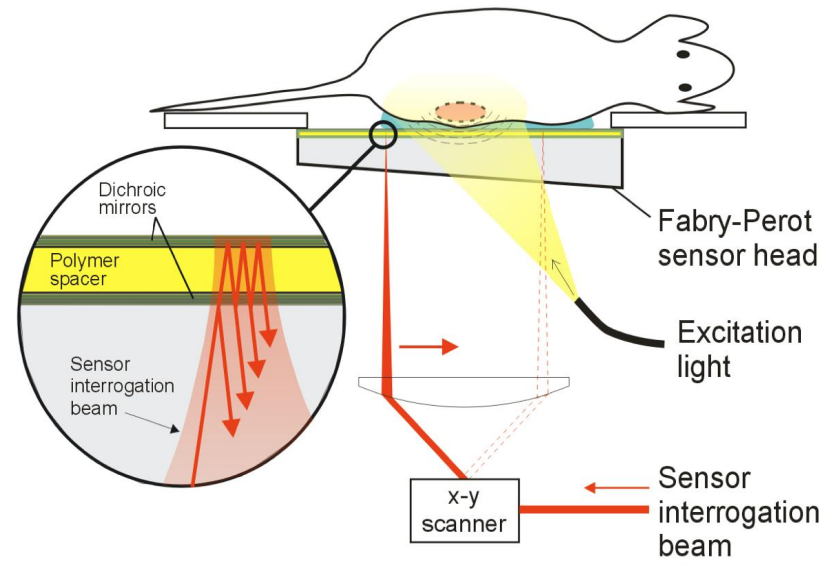

Figure 1: FP sensor based PA imaging system (figure adapted from [6]).

The transduction mechanism of the FP sensor [7] is one in which an incident acoustic wave modulates its optical thickness resulting in a corresponding modulation in its reflectivity. The latter is detected using a continuous wave interrogation laser beam focussed on the plane of the sensor. The advantage of this approach to ultrasonic detection over conventional piezoelectric detectors is that it provides a highly uniform frequency response (typical $-3 \mathrm{~dB}$ bandwidths of 20-40 MHz) and a wide directivity due to the small $(<\varnothing 100 \mu \mathrm{m})$ acoustic element size, both of which impact directly upon PA image quality. The sensitivity in terms of noise equivalent pressure (NEP) is in the range

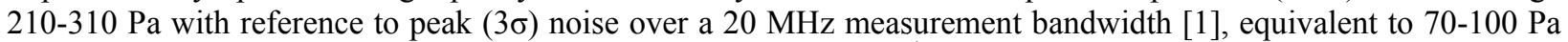
over $20 \mathrm{MHz}$ for root mean squared (RMS) noise or $15-23 \mathrm{mPa} / \sqrt{ } \mathrm{Hz}$. To put this into perspective a piezoelectric sensor of comparable element size and frequency response would have $>2$ orders-of-magnitude poorer NEP [1].

Photons Plus Ultrasound: Imaging and Sensing 2017, edited by Alexander A. Oraevsky, Lihong V. Wang, Proc. of SPIE Vol. 10064, 100641V · (c) 2017 SPIE · CCC code: 1605-7422/17/\$18 · doi: 10.1117/12.2249993 
With these characteristics the FP system provides high resolution, high fidelity PA images of tissue with a maximum penetration depth of $\approx 10 \mathrm{~mm}$ [8]. There is strong motivation to increase this maximum depth as it would significantly extend the range of available preclinical and clinical applications. This requires a significant improvement in NEP but this is limited by the divergent nature of the sensor interrogation beam which encounters beam walk-off inside the FP spacer [9]-[11] as shown in figure 2a; the effect of beam-walk is to limit the cavity finesse and therefore the sensitivity.

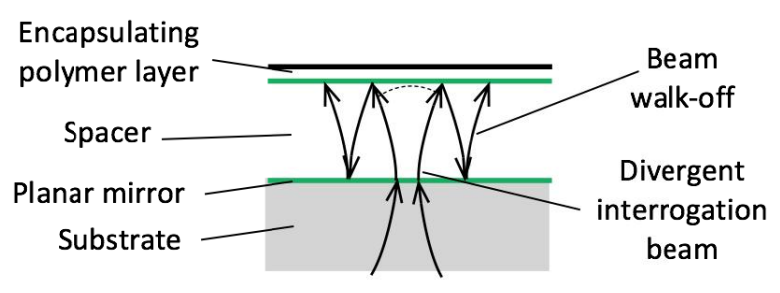

(a)

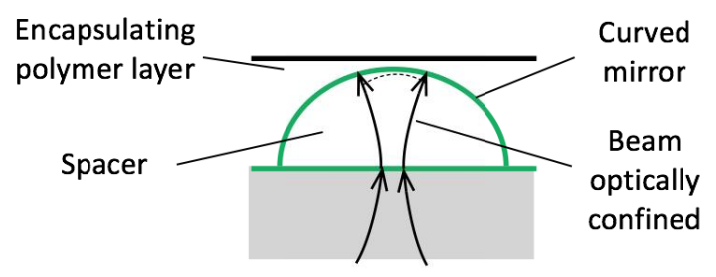

(b)

Figure 2: Schematics of (a) planar FP and (b) planoconcave microresonator sensor illustrating beam walk-off in the former and its elimination in the latter.

To overcome this limitation, planoconcave optical microresonator sensors were developed with a curved top mirror designed to refocus light upon each round trip in the spacer, thereby eliminating beam walk-off and improving optical confinement [9]-[11] as illustrated in figure $2 \mathrm{~b}$. With this design it was found that NEP could be improved significantly by increasing the mirror reflectance and spacer thickness [11], the latter at a cost to bandwidth as the sensor responds to the spatial integral of the acoustic field. This trade-off enables a family of sensors with different bandwidths and sensitivities to be fabricated. For example, we have previously developed a $100 \mu \mathrm{m}$ thick sensor with a $9 \mathrm{MHz}-3 \mathrm{~dB}$ bandwidth and NEP $<10 \mathrm{~Pa}(3.2 \mathrm{mPa} / \sqrt{\mathrm{Hz}})$ and a $340 \mu \mathrm{m}$ thick sensor with a $3 \mathrm{MHz}-3 \mathrm{~dB}$ bandwidth and NEP $<3 \mathrm{~Pa}(1.6 \mathrm{mPa} / \sqrt{ } \mathrm{Hz})$ [11]. The latter represents an order of magnitude improvement in NEP over the planar FP sensor. In addition to higher sensitivity the microresonators have the same frequency response and directivity as the FP sensor as they are acoustically identical [11]. This is because both types of sensor are embedded in a planar polymer layer (figure 2) with mirrors that are ultrasonically sub-wavelength and therefore acoustically negligible.

These characteristics suggest that when used for PA imaging the planoconcave microresonator sensors will provide near identical image quality as the planar FP sensor but with significantly greater penetration depth. This paper examines this experimentally. Two experiments are described, the first investigating penetration depth and the second testing image quality. The results demonstrate that planoconcave microresonator sensors achieve deeper imaging with no loss in image quality.

\section{IMAGING PENETRATION DEPTH}

To investigate the achievable penetration depth for PA imaging in tomography mode using microresonator sensors, a tissue phantom was imaged in forward mode. Figure 3 shows a schematic of the experimental setup.

The phantom consisted of 8 dye-filled polythene tubes arranged vertically in a liquid scattering medium composed of $0.8 \%$ Intralipid in deionised (DI) water. At $1064 \mathrm{~nm}$ the medium had $\mu_{\mathrm{a}}=0.12 \mathrm{~cm}^{-1}$ and $\mu_{\mathrm{s}}{ }^{\circ}=6 \mathrm{~cm}^{-1}$, i.e. $\mu_{\text {eff }}=1.5 \mathrm{~cm}^{-1}$; an attenuation comparable to soft tissues at $750 \mathrm{~nm}$ [12]. The tubes had an inner and outer diameter of $580 \mu \mathrm{m}$ and $960 \mu \mathrm{m}$ respectively. They contained an Indian ink solution of $\mu_{\mathrm{a}}=3 \mathrm{~cm}^{-1}$ (at $1064 \mathrm{~nm}$ ); comparable to $90 \%$ oxygenated blood at $750 \mathrm{~nm}$.

The excitation source was a fibre-coupled $1064 \mathrm{~nm}$ Q-switched ND:YAG laser (Minilite, Continuum Lasers) with a pulse repetition frequency (PRF) of $20 \mathrm{~Hz}$ and a pulse-width of 6ns. The pulse energy at the fibre output was 14 $\mathrm{mJ}$. The beam was expanded to $\varnothing 10 \mathrm{~mm}$ yielding a surface fluence of $18 \mathrm{~mJ} / \mathrm{cm}^{2}$ below the maximum permissible exposure for human skin [13].

The phantom was imaged using a $250 \mu \mathrm{m}$ thick microresonator sensor with a $-3 \mathrm{~dB}$ bandwidth of $4 \mathrm{MHz}$ and an NEP of $2 \mathrm{mPa} / \sqrt{\mathrm{Hz}}$. The sensor was fixed in position and the phantom was mechanically scanned in $2 \mathrm{D}$ so as to emulate a 2D array of identical sensors imaging a fixed phantom. Acoustic waveforms were acquired by an oscilloscope (TDS5K, Tektronix) triggered by a photodiode. The total scan area was $41 \mathrm{~mm}$ by $12 \mathrm{~mm}$, scanned in 
steps of $100 \mu \mathrm{m}$ and $200 \mu \mathrm{m}$. No signal averaging was used. The microresonator sensor was then replaced by an FP sensor for comparison and the scan was repeated.

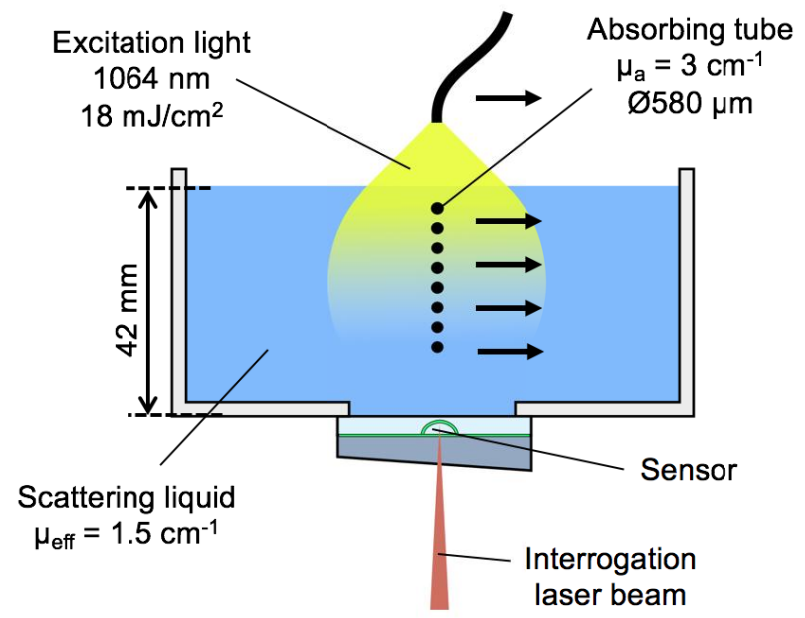

Figure 3: Experimental phantom imaging setup.

3D Images were reconstructed by time reversal using K-wave [14]. Prior to reconstruction waveforms were filtered with a low pass filter with a $-3 \mathrm{~dB}$ cut-off equal to the $-3 \mathrm{~dB}$ bandwidth of the sensor. Reconstructed images were subjected to a 1D fluence correction [15] to aid visualisation. 2D cross-sections through the images are plotted in figure 4.

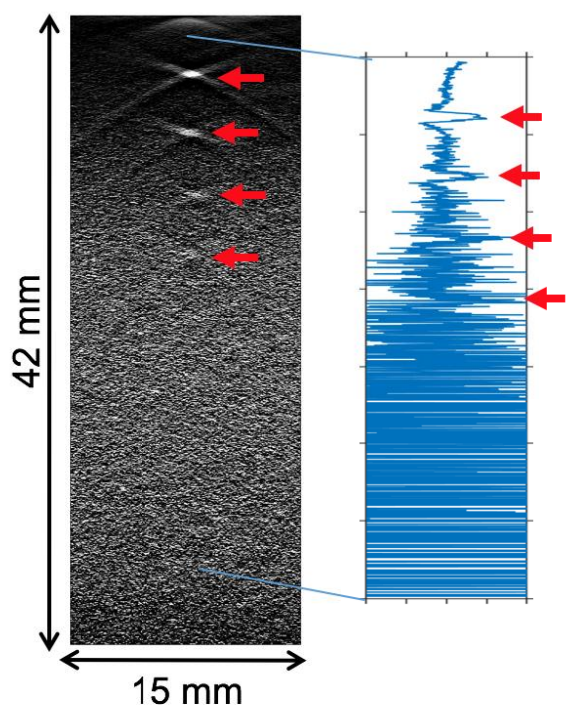

(a)

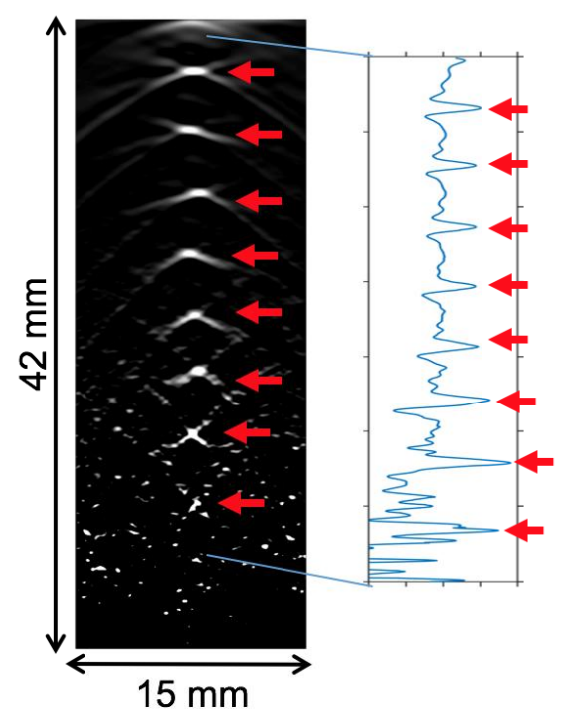

(b)

Figure 4: 2D cross-sections and vertical profiles through 3D PA images of a tube phantom (figure 3) acquired with (a) an FP sensor and (b) a planoconcave microresonator sensor. Arrows indicate tubes.

Compared to typical PA images obtained with the FP sensor both images show significant artefacts. These are a consequence of the experimental conditions; a combination of limited-aperture [16] and tube-related artefacts due to the acoustic mismatch of the tubes to the background. The latter do not appear in vivo and the former can be removed by increasing the size of the scan area which was limited here by the mechanical scanning setup.

In the FP planar sensor image, only the top 3 tubes are clearly visible and the $4^{\text {th }}$ is just visible above the evident noise floor. By contrast in the microresonator image all 8 tubes can be seen. This represents an increase of $16 \mathrm{~mm}$ in imaging depth. 


\section{IMAGE QUALITY}

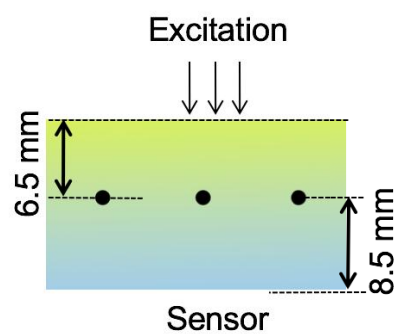

Figure 5: Schematic of second phantom.

To assess image quality a second phantom was imaged with a $100 \mu \mathrm{m}$ microresonator. The phantom (figure 5) was $15 \mathrm{~mm}$ deep with tubes made of THV of 604 (725) $\mu \mathrm{m}$ inner (outer) diameter. As above, the imaging experiment was repeated using the planar FP sensor for comparison. The results are shown in figure 6.

Planar sensor image
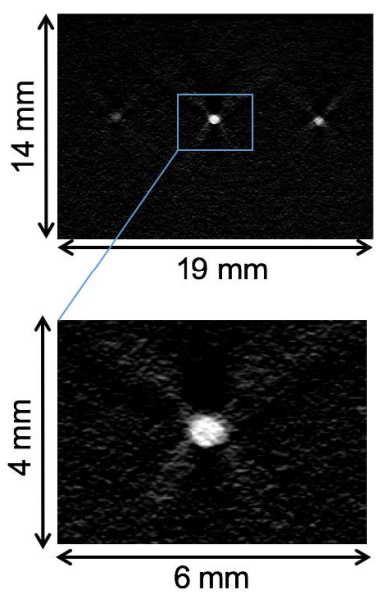

$100 \mu \mathrm{m}$ microres. image
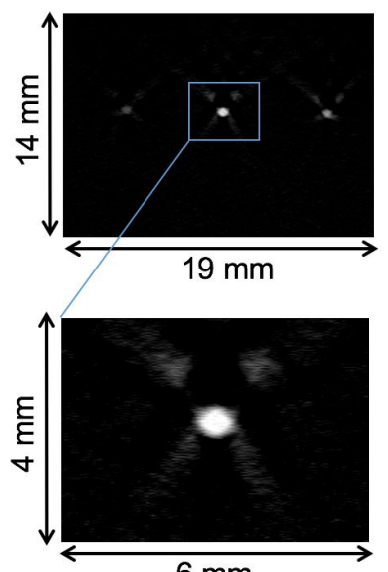

$6 \mathrm{~mm}$
Lateral profiles
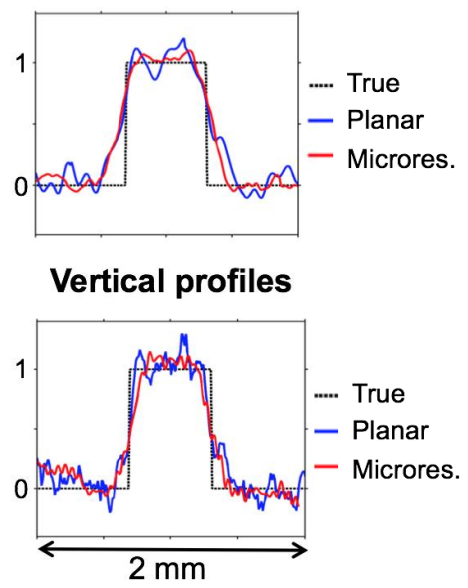

Figure 6: Experimental phantom images obtained using the FP sensor and a $100 \mu \mathrm{m}$ microresonator sensor. The zoomed in images show the middle tube in higher detail. Lateral and vertical profiles are plotted taken through the centre of the middle tube.

The images are relatively artefact-free and provide a sharp, faithful representation of the three tubes. The microresonator image is practically indistinguishable from that of the FP with the only apparent difference being a better SNR due to the higher sensitivity. The profiles through the centre of the middle tube show no noticeable differences and are the expected width given the known size of the tubes.

\section{CONCLUSIONS}

In summary, tomographic PA images obtained by planoconcave microresonator sensors were presented. As compared to the well-established planar FP sensor, the microresonator sensors achieved $16 \mathrm{~mm}$ deeper imaging in a tissue-realistic phantom, with no loss in image quality. These sensors therefore offer the prospect of in vivo imaging with high resolution and fidelity and considerably greater $(2-3 \mathrm{~cm})$ penetration depth.

Imaging was performed with a single sensor emulating a $2 \mathrm{D}$ array but in the future it is anticipated that $2 \mathrm{D}$ arrays will be fabricated using techniques such as nano-imprinting. In conjunction with multi-beam interrogation schemes [17] and sub-sampling approaches it will then be possible to perform in vivo imaging in a reasonable time frame (on the scale of seconds). 
It may be also possible to increase sensitivity and penetration depth still further. For instance by fabricating sensors using polymers of lower optical absorption to improve finesse [9] or of lower Young's modulus to increase compliance. This would allow even deeper imaging and enable still more challenging applications such as the noninvasive clinical assessment of breast tumours.

\section{ACKNOWLEDGMENTS}

This work was funded by EPSRC and European Union project FAMOS (FP7 ICT, Contract 317744).

\section{REFERENCES}

[1] E. Zhang, J. Laufer, and P. Beard, "Backward-mode multiwavelength photoacoustic scanner using a planar Fabry-Perot polymer film ultrasound sensor for high-resolution three-dimensional imaging of biological tissues," Appl. Opt., vol. 47, no. 4, pp. 561-577, Mar. 2008.

[2] H. Li, B. Dong, Z. Zhang, H. F. Zhang, and C. Sun, "A transparent broadband ultrasonic detector based on an optical micro-ring resonator for photoacoustic microscopy.," Sci. Rep., vol. 4, p. 4496, Jan. 2014.

[3] A. Rosenthal, D. Razansky, and V. Ntziachristos, "High-sensitivity compact ultrasonic detector based on a pi-phaseshifted fiber Bragg grating.," Opt. Lett., vol. 36, no. 10, pp. 1833-5, May 2011.

[4] R. Nuster, P. Slezak, and G. Paltauf, "High resolution three-dimensional photoacoutic tomography with CCD-camera based ultrasound detection," Biomed. Opt. Express, vol. 5, no. 8, p. 2635, Jul. 2014.

[5] S. M. Maswadi, B. L. Ibey, C. C. Roth, D. A. Tsyboulski, H. T. Beier, R. D. Glickman, and A. A. Oraevsky, “All-optical optoacoustic microscopy based on probe beam deflection technique," Photoacoustics, vol. 4, no. 1, pp. 91-101, 2016.

[6] J. Laufer, P. Johnson, E. Zhang, B. Treeby, B. Cox, B. Pedley, and P. Beard, "In vivo preclinical photoacoustic imaging of tumor vasculature development and therapy," J. Biomed. Opt., vol. 17, no. 5, pp. 56016-1-56016-8, May 2012.

[7] P. C. Beard, F. Perennes, and T. N. Mills, "Transduction mechanisms of the Fabry-Perot polymer film sensing concept for wideband ultrasound detection," IEEE Trans. Ultrason. Ferroelectr. Freq. Control, vol. 46, no. 6, pp. 1575-1582, Jan. 1999.

[8] A. P. Jathoul, J. Laufer, O. Ogunlade, B. Treeby, B. Cox, E. Zhang, P. Johnson, A. R. Pizzey, B. Philip, T. Marafioti, M. F. Lythgoe, R. B. Pedley, M. a. Pule, and P. Beard, "Deep in vivo photoacoustic imaging of mammalian tissues using a tyrosinase-based genetic reporter," Nat. Photonics, vol. 9, no. April, pp. 239-246, Mar. 2015.

[9] J. A. Guggenheim, E. Z. Zhang, and P. C. Beard, "Planoconcave optical microresonator sensors for photoacoustic imaging: pushing the limits of sensitivity (Conference Presentation)," Proc. SPIE, vol. 9708. p. 97082M-97082M-1, 2016.

[10] J. A. Guggenheim, J. Li, E. Z. Zhang, and P. C. Beard, "Frequency response and directivity of highly sensitive optical microresonator detectors for photoacoustic imaging," in Proc. of SPIE, Photons Plus Ultrasound: Imaging and Sensing, 2015, vol. 9323, p. 93231C.

[11] J. A. Guggenheim, J. Li, T. J. Allen, R. J. Colchester, S. Noimark, O. Ogunlade, I. P. Parkin, I. Papakonstantinou, A. Desjardins, E. Z. Zhang, and P. C. Beard, "Ultra-sensitive planoconcave optical microresonators for ultrasound sensing," p. In review.

[12] S. L. Jacques, “Optical properties of biological tissues: a review,” Phys. Med. Biol., vol. 58, no. 14, pp. 5007-5008, Jul. 2013.

[13] C. Li and L. V Wang, "Photoacoustic tomography and sensing in biomedicine," Phys. Med. Biol., vol. 54, no. 19, pp. R59-97, Oct. 2009.

[14] B. E. Treeby and B. T. Cox, "k-Wave: MATLAB toolbox for the simulation and reconstruction of photoacoustic wave fields," J. Biomed. Opt., vol. 15, no. 2, p. 21314, 2010.

[15] B. E. Treeby, J. Jaros, and B. T. Cox, "Advanced photoacoustic image reconstruction using the k-Wave toolbox," in Proceedings of SPIE, 2016, vol. 9708, p. 97082P.

[16] R. Ellwood, O. Ogunlade, E. Zhang, P. Beard, and B. Cox, "Photoacoustic tomography using orthogonal Fabry-Pérot sensors," J. Biomed. Opt., vol. 22, no. 4, p. 41009, 2016.

[17] N. Huynh, O. Ogunlade, E. Zhang, B. Cox, and P. Beard, "Photoacoustic imaging using an 8-beam Fabry-Perot scanner," in Proceedings of SPIE, 2016, vol. 9708, p. 97082L. 